\title{
CONFORMAL MAPPINGS AND THE DISTORTION OF MODULI OF RECTANGLES
}

\author{
BRUCE PALKA 1
}

ABSTRACT. In this paper it is shown that for an orientation-preserving homeomorphism $f$ between domains $D$ and $D^{\prime}$ in $\bar{C}$ to be conformal it is necessary and sufficient that there is an $a>1$ such that $\bmod f(Q)=\bmod Q$ for each square $Q$ in $D$ and for each oriented rectangle $Q$ in $D$ with $\bmod Q=a$.

1. Quadrilaterals. Let $Q$ be a Jordan domain in $\overline{\mathbf{C}}$, the extended complex plane, and denote its boundary by $\partial Q$. Let $z_{1}, z_{2}, z_{3}, z_{4}$ be distinct points of $\partial Q$ such that traversing $\partial Q$ by starting at $z_{1}$ and passing through $z_{2}, z_{3}$ and $z_{4}$ in order describes the positive orientation of $\partial Q$ with respect to $Q$. We call such a configuration a quadrilateral with vertices $z_{1}, z_{2}, z_{3}, z_{4}$ and denote it by $Q\left(z_{1}, z_{2}, z_{3}, z_{4}\right)$-or simply by $Q$, when no confusion about the vertices can arise. If $Q=Q\left(z_{1}, z_{2}, z_{3}, z_{4}\right)$ is a quadrilateral, we write $Q^{*}=$ $Q^{*}\left(z_{1}, z_{2}, z_{3}, z_{4}\right)$ for the conjugate quadrilateral:

$$
Q^{*}\left(z_{1}, z_{2}, z_{3}, z_{4}\right)=Q\left(z_{2}, z_{3}, z_{4}, z_{1}\right) .
$$

We use "rectangle" to mean a quadrilateral $Q\left(z_{1}, z_{2}, z_{3}, z_{4}\right)$, where $Q$ is a finite rectangle and the $z_{j}$ are actual vertices of $Q$. A rectangle is said to be oriented if its sides are pairwise parallel to the real and imaginary axes. Similarly, we use "square" to refer to a finite square and its actual vertices.

If $Q=Q\left(z_{1}, z_{2}, z_{3}, z_{4}\right)$ is a quadrilateral there is a unique $M>0$ with the property that $R=\{z \mid 0 \leq \operatorname{Re} z \leq M, 0 \leq \operatorname{Im} z \leq 1\}$ can be mapped homeomorphically onto $\bar{Q}$ by a map $\phi$ which is conformal in the interior of $R$ and satisfies $\phi(0)=z_{1}, \phi(M)=z_{2}, \phi(M+i)=z_{3}, \phi(i)=z_{4}$. The number $M$ is called the modulus of $Q$, written $M=\bmod Q$. It is easy to verify that

$$
\bmod Q \cdot \bmod Q^{*}=1 \text {. }
$$

Received by the editors February $11,1974$.

AMS (MOS) subject classifications (1970). Primary 30A30, 30A60.

Key words and phrases. Conformal mapping, quasiconformal mapping, modulus of a quadrilateral.

1 The author acknowledges support under NSF Grant GP-28574. 
2. Conformal mappings and quadrilaterals. Let $D$ be a domain in $\overline{\mathbf{C}}$. If $Q$ is a quadrilateral with $\bar{Q} \subset D$ we say $Q$ is a quadrilateral in $D$. Let $f$ be an orientation-preserving homeomorphism of $D$ onto a domain $D^{\prime}$. If $Q=Q\left(z_{1}, z_{2}, z_{3}, z_{4}\right)$ is a quadrilateral in $D$,

$$
f(Q)=f(Q)\left(f\left(z_{1}\right), f\left(z_{2}\right), f\left(z_{3}\right), f\left(z_{4}\right)\right)
$$

is a quadrilateral in $D^{\prime}$. It is not difficult to show that $f$ is a conformal mapping if and only if

$$
\bmod f(Q)=\bmod Q
$$

for each quadrilateral $Q$ in $D$. It is natural to ask whether one can infer the conformality of $f$ by knowing that (2) holds for some restricted class of quadrilaterals. The answer is, of course, affirmative (see [3]). The known results in this direction lead one to the tempting

(3) Conjecture. If (2) is satisfied for each square $Q$ in $D$, then $f$ is a conformal mapping. (Note: for a square $Q, \bmod Q=1$. )

Whether (3) is true or not remains an open question. It is known that $f$ need not be conformal if (2) is required to hold only for each oriented square in D. (See [3] for a counterexample.) The difficulty in establishing (3) seems to derive from having "too much symmetry" in the hypothesis. In this paper we show that the introduction of an element of "asymmetry" is sufficient to force the conformality of $f$. We observe at the outset that (2) holds for a particular square $Q$ in $D$ if and only if either

$$
\bmod f(Q) \leq \bmod Q
$$

or

$$
\bmod f(Q) \geq \bmod Q
$$

This follows from (1) and the fact that for any quadrilateral $Q$ in $D$

$$
f\left(Q^{*}\right)=f(Q)^{*}
$$

3. Lemmas. For the remainder of this paper we assume that $D$ and $D^{\prime}$ are domains in $\overline{\mathbf{C}}$ and that $f$ is an orientation-preserving homeomorphism of $D$ onto $D^{\prime}$. We begin with two elementary lemmas.

Lemma 1. Let $K \geq 1, a>0$, and assume that $\bmod f(Q) \leq K a$ for each rectangle (resp. oriented rectangle) $Q$ in $D$ with $\bmod Q=a$. Let $Q$ be a rectangle (resp. oriented rectangle) in $D$ with $\bmod 2=1 / a$. Then $\bmod f(Q) \geq 1 / K a$. 
Proof. $Q^{*}$ is a rectangle (resp. oriented rectangle) and $\bmod Q^{*}=a$, whence $\bmod f\left(Q^{*}\right) \leq K a$. The result follows from (6) and (1).

Lemma 2. Let $K \geq 1, a>0$, and assume that $\bmod f(Q) \leq K a$ for each rectangle (resp. oriented rectangle) $Q$ in $D$ with $\bmod Q=a$. Let $Q$ be a rectangle (resp. oriented rectangle) in $D$ with $\bmod Q=b \leq a$. Then $\bmod f(Q) \leq 2 K b$.

Proof. We may assume that $b<a$ and that $Q$ has vertices $z_{1}=0$, $z_{2}=b, z_{3}=b+i, z_{4}=i$. Let $n$ be the positive integer such that $n b \leq a<$ $(n+1) b$. The segments $L_{p}: 0 \leq \dot{x} \leq b, y=p b / a$ for $p=1, \cdots, n$ divide $Q^{*}$ into rectangles $Q_{1}^{*}, \cdots, Q_{n}^{*}$ and, in the case $n t<a$, a rectangle $Q_{n+1}^{*}$, where $\bmod Q_{p}^{*}=1 / a$ for $p=1, \cdots, n$.
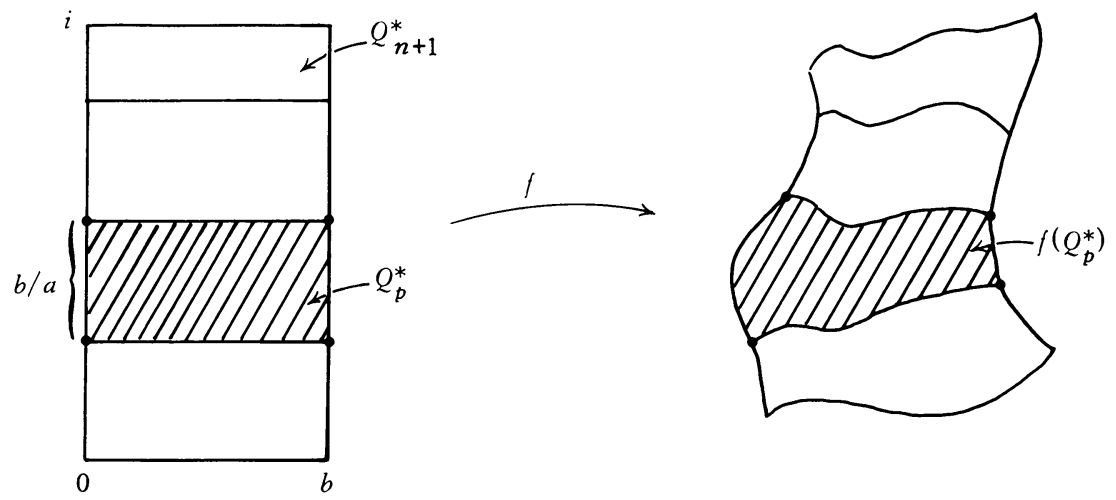

\section{Figure 1}

By $[5$, Lemma 4.2 , p. 26] and Lemma 1 ,

$$
\bmod f\left(Q^{*}\right) \geq \sum_{p=1}^{n} \bmod f\left(Q_{p}^{*}\right) \geq \frac{n}{K a}
$$

Utilizing (6) and (1) we obtain

$$
\bmod f(Q) \leq \frac{K a}{n}=\frac{n+1}{n} \frac{K a}{n+1} \leq 2 K b
$$

as asserted.

Remark 1. If in Lemma 2 we have $b=a / n, n$ a positive integer, the above proof shows that $\bmod f(Q) \leq K b$.

To establish the main lemma in this paper we require a result related to the Ahlfors distortion theorem. This result, certainly familiar to those in the field of conformal mapping, has apparently never been published in the form in which we require it. 
Lemma 3. Let $G=\{z \mid 0 \leq \operatorname{Im} z \leq 1\}$ and let $Q=Q\left(z_{1}, z_{2}, z_{3}, z_{4}\right)$ be a quadrilateral with $\bar{Q} \subset G$ and $\operatorname{Re} z_{1}<\operatorname{Re} z_{2}, \operatorname{Re} z_{4}<\operatorname{Re} z_{3}, \operatorname{Im} z_{1}=\operatorname{Im} z_{2}$ $=0, \operatorname{Im} z_{3}=\operatorname{Im} z_{4}=1$ such that $\partial Q$ consists of the line segment joining $z_{1}$ to $z_{2}$, an arc $\beta$ joining $z_{2}$ to $z_{3}$, the line segment joining $z_{3}$ to $z_{4}$ and an arc a joining $z_{4}$ to $z_{1}$. Assume that $\bmod 2 \geq 1$. Then

$$
d=\inf _{z \in \beta} \operatorname{Re} z-\sup _{z \in a} \operatorname{Re} z>0 .
$$

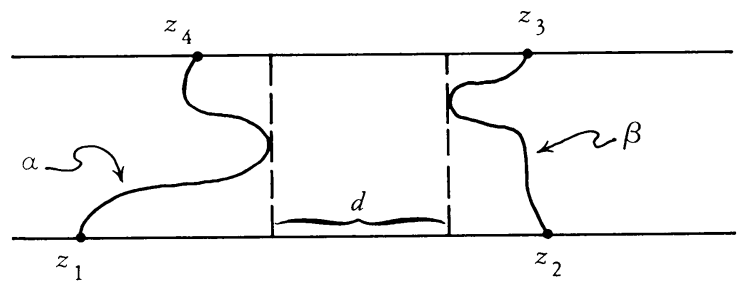

Figure 2

Proof. Let $\bmod Q=M \geq 1$ and let $\phi$ be the homeomorphism of $R=$ $\{z \mid 0 \leq \operatorname{Re} z \leq M, 0 \leq \operatorname{Im} z \leq 1\}$ onto $\bar{Q}$ used in the definition of modulus. We may assume that $\inf _{z \in \alpha}$ Re $z=0$. The map $b$, defined in $E=\{z|1<| z \mid<$ $\left.e^{\pi M}, \operatorname{Im} z>0\right\}$ by $b(z)=\exp \left\{\pi \phi\left(\pi^{-1} \log z\right)\right\}$, where the principal branch of the logarithm is used, can be extended by reflection to a conformal mapping of the annulus $A=\left\{z|1<| z \mid<e^{\pi M}\right\}$ onto a ring domain $A^{\prime}$ with complementary components $C_{0}$ and $C_{1}$ in $\overline{\mathbf{C}}$, where $0 \in C_{0}$ and $\infty \in C_{1}$. Thus $\bmod A^{\prime}$ $=\pi M$. (By definition, the modulus of a ring domain $A^{\prime}$ with nondegenerate complementary components is $\log \rho$, where $\rho>1$ is the unique number such that $A^{\prime}$ can be mapped conformally onto $A=\{z|1<| z \mid<\rho\}$.) If $d \leq 0$ there is a number $r>0$ such that both $C_{0}$ and $C_{1}$ meet $S=\{z|| z \mid=r\}$. By a result of Teichmüller $\left[6\right.$, p. 638] this would imply that $\bmod A^{\prime}<\pi$-strict inequality holding, since $A^{\prime}$ is not of the extremal type described by Teichinüller's theorem-hence that $M<1$, contrary to hypothesis. We conclude that $d>0$, as desired.
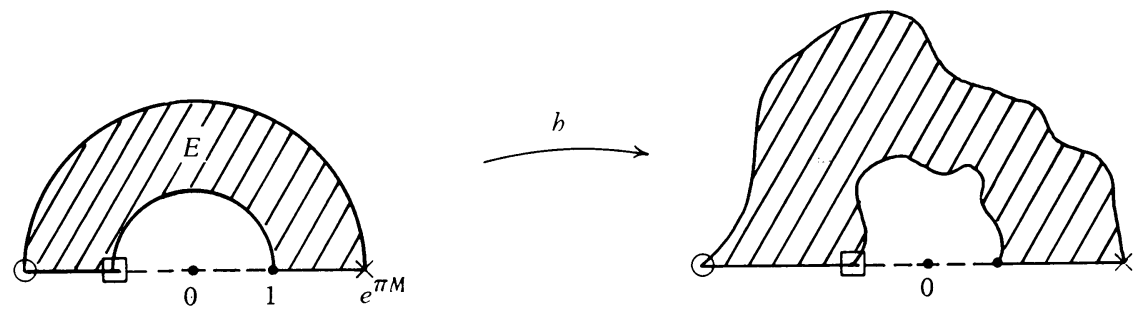

Figure 3 
We now turn to the main lemma in this paper.

Lemma 4. Let $a>1$ and assume that (4) is satisfied for each oriented square $Q$ in $D$ and each oriented rectangle $Q$ in $D$ with $\bmod Q=a$. Let $Q$ be an oriented rectangle in $D$ with $\bmod Q=m, m$ a positive integer. Then $\bmod f(Q) \leq K m$, where $K$ depends only on a.

Proof. If $m \leq a, \bmod f(Q) \leq 2 m$ by Lemma 2 . We assume that $m>a$. We may further assume that $Q$ has vertices $z_{1}=0, z_{2}=m, z_{3}=m+i, z_{4}=i$ and, following $f$ by a conformal map, that $f(Q)$ is a rectangle with vertices $f\left(z_{1}\right)=0, f\left(z_{2}\right)=M, f\left(z_{3}\right)=M+i, f\left(z_{4}\right)=i$, where $M=\bmod f(Q)$.

Fix a positive integer $j$ satisfying

$$
1+1 / j \leq a
$$

Denote by $L_{p}$ the segment $L_{p}: x=p / j, 0 \leq y \leq 1$, for $p=0,1, \cdots, m j$ and write $L_{p}^{\prime}=f\left(L_{p}\right)$. For $p=0,1, \cdots,(m-1) j$ let $Q_{p}$ be the square with vertices $z_{1}=p / j, z_{2}=1+p / j, z_{3}=1+p / j+i, z_{4}=p / j+i$. Since $\bmod f\left(Q_{p}\right)=1, f\left(Q_{p}\right)$ is a quadrilateral satisfying the hypotheses of Lemma 3 , whence $\alpha_{p}=L_{p}^{\prime}$ and $\beta_{p}=L_{p+j}^{\prime}$ can be separated in $f\left(Q_{p}\right)$ by a vertical segment $\lambda_{p}$ joining the horizontal sides of $f(Q)$. We choose the segments $\lambda_{p}$ so that $\lambda_{p+1}$ is to the right of $\lambda_{p}$ for $p=0, \cdots,(m-1) j-1$. The segments $\lambda_{p}$ divide $f(Q)$ into rectangles $R_{l}^{\prime}, l=0,1, \cdots,(m-1) j+1$, where $R_{0}^{\prime}$ is bounded on the left by $L_{0}^{\prime}, R_{(m-1) j+1}^{\prime}$ is bounded on the right by $L_{m j}^{\prime}$, and, for $l=1, \cdots,(m-1) j, R_{l}^{\prime}$ is bounded on the left by $\lambda_{l-1}$ and on the right by $\lambda_{l}$.
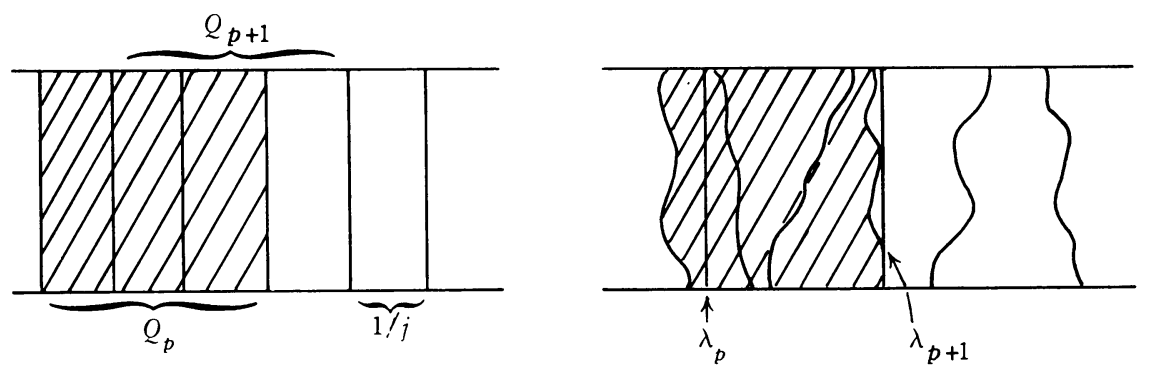

Figure 4

Now $R_{0}^{\prime} \subset f\left(Q_{0}\right)$ and by $[5$, Lemma 4.2, p. 26],

$$
\bmod R_{0}^{\prime} \leq \bmod f\left(Q_{0}\right)=1<a
$$

Similarly, 


$$
\bmod R_{(m-1) j+1}^{\prime} \leq a .
$$

For $l=1, \cdots,(m-1) j, R_{l}^{\prime} \subset f\left(R_{l}\right)$, where $R_{l}$ is the rectangle with vertices $z_{1}=(l-1) / j, z_{2}=1+l / j, z_{3}=1+l / j+i, z_{4}=(l-1) / j+i$. Now $\bmod R_{l}=$ $1+1 / j \leq a$, so again invoking [5, Lemma 4.2, p. 26], as well as Lemma 2 and (7), we infer

$$
\bmod R_{l}^{\prime} \leq \bmod f\left(R_{l}\right) \leq 2 \bmod R_{l} \leq 2 a .
$$

Combining (8), (9) and (10) we conclude

$$
\bmod f(Q)=\sum_{l=0}^{(m-1) j+1} \bmod R_{l}^{\prime} \leq a+2(m-1) j a+a<2 a(j+1)_{m}
$$

The result follows with $K=2 a(j+1)$.

4. Main results. We can now state

Theorem 1. Let $a>1$ and assume that (4) is satisfied for each square $Q$ in $D$ and for each oriented rectangle $Q$ in $D$ with $\bmod Q=a$. Then $f$ is a conformal mapping.

Proof. Let $m$ be a positive integer. By Lemma 4 , $\bmod f(Q) \leq K m$ for each oriented rectangle $Q$ in $D$ with $\bmod Q=m$, where $K$ depends only on a. From Lemma 2 it follows that $\bmod f(Q) \leq 2 K \bmod Q$ for each oriented rectangle $Q$ in $D$ with mod $Q \leq m$. Since $m$ is arbitrary, we conclude that nod $f(Q) \leq 2 K \bmod Q$ for each oriented rectangle $Q$ in $D$ and, hence, that $f$ is a quasiconformal mapping [3, Theorem 2]. However, since (4) holds for each square in $D, f$ is in fact a conformal mapping [5, Theorem 9.3, p. 52].

Corollary 1. Let $a>1$ be an integer and assume that (4) is satisfied for each rectangle $Q$ in $D$ with $\bmod Q=a$. Then $f$ is a conformal mapping.

Proof. From Remark 1 we infer that (4) is satisfied for each rectangle $Q$ in $D$ with $\bmod Q=a / a=1$, i.e. for each square $Q$ in $D$. Theorem 1 now applies.

Remark 2. Theorem 1 and its corollary have obvious analogues in terms of (5).

Remark 3. With an added hypothesis on the size of $a$ the above results can be extended with little modification in the proofs to the quasiconformal case. We have as yet been unable to show that $f$ is $K$-quasiconformal if 
$\bmod f(Q) \leq K \bmod Q$ for each square $Q$ in $D$ and for each oriented rectangle $Q$ with $\bmod Q=a>1$. We can show that $f$ is $K$-quasiconformal if the above holds with $a>K+1$.

\section{REFERENCES}

1. C. A. Cazacu, Sur les inégalités de Rengel et la définition géométrique des représentations quasi-conformes, Rev. Romaine Math. Pures Appl. (9) 9 (1964), 141-155. MR. 31 \#4904.

2. F. W. Gehring, The definitions and exceptional sets for quasi-conformal mappings, Ann. Acad. Sci. Fenn. Ser. A I No. 281 (1960), 28pp. MR 23 \# A1800.

3. F. W. Gehring and J. Väisälä, On the geometric definition for quasiconformal mappings, Comment. Math. Helv. 36 (1961), 19-32. MR 25 \#4099.

4. J. A. Kelingos, Contributions to the theory of quasiconformal mappings, Thesis, University of Michigan, Ann Arbor, Mich., 1963.

5. O. Lehto and K. I. Virtanen, Quasikonforme Abbildungen, Die Grundlehren der math. Wissenschaften in Einzeldarstellungen mit besonderer Berücksichtigung der Anwendungsgebiete, Band 126, Springer-Verlag, Berlin and New York, 1965. MR 32 \#5872.

6. O. Teichmüller, Untersuchungen über konforme und quasikonforme Abbildung, Deutsche Math. 3 (1938), 621-678.

DEPARTMENT OF MATHEMATICS, BROWN UNIVERSITY, PROVIDENCE, RHODE ISL AND 02912

Current address : Department of Mathematics, University of Texas at Austin, Austin, Texas 78712 